\title{
Melatonin Receptors Agonistic Activities of Phenols from Gastrodia elata
}

\author{
Si-Yue Chen ${ }^{1,2} \cdot$ Chang-An Geng $^{1} \cdot$ Yun-Bao Ma ${ }^{1} \cdot J^{-}-J u n$ Chen $^{1,2}$ (D)
}

Received: 23 April 2019 / Accepted: 22 May 2019 / Published online: 7 June 2019

(c) The Author(s) 2019

\section{Abstract}

Gastrodia elata is a famous traditional Chinese herb with medicinal and edible application. In this study, three new polybenzyls, gastropolybenzylols G-I (1-3) were isolated from the EtOAc extract of G. elata. Their structures were identified by extensive spectroscopic analyses involving HRESIMS, UV, IR, 1D and 2D NMR. Compound 1 showed agonistic effects on $\mathrm{MT}_{1}$ and $\mathrm{MT}_{2}$ receptors with agonistic rates of $55.91 \pm 4.84 \%$ and $165.13 \pm 5.65 \%$ at the concentration of $0.5 \mathrm{mM}$, respectively, and an $\mathrm{EC}_{50}$ value of $76.24 \mu \mathrm{M}$ on $\mathrm{MT}_{2}$ receptor.

\section{Graphic Abstract}

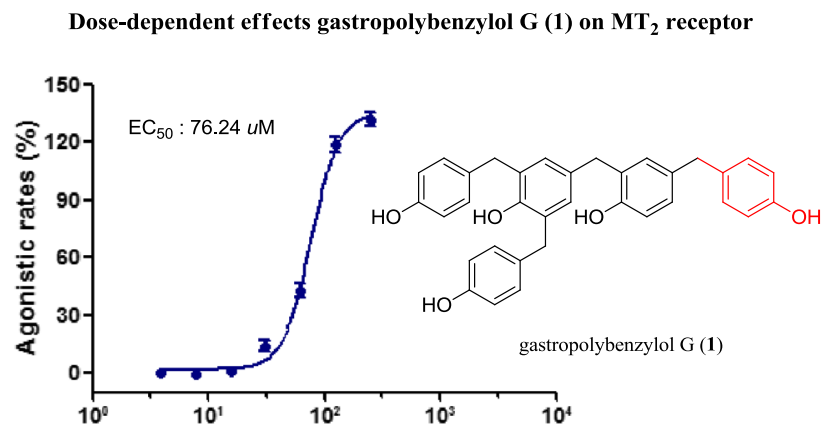

Keywords Gastrodia elata $\cdot$ Gastropolybenzylols · Melatonin receptors

Electronic supplementary material The online version of this article (https://doi.org/10.1007/s13659-019-0213-2) contains supplementary material, which is available to authorized users.

Ji-Jun Chen

chenjj@mail.kib.ac.cn

1 State Key Laboratory of Phytochemistry and Plant Resources in West China, Kunming Institute of Botany, Yunnan Key Laboratory of Natural Medicinal Chemistry, Chinese Academy of Sciences, Kunming 650201, China

2 University of Chinese Academy of Sciences, Beijing 100049, China

\section{Introduction}

Gastrodia elata, a saprophytic herb from orchid family, mainly distributed in East Asia, Southeast Asia and Oceania. The main constituents of G. elata are phenolics, polysaccharides, sterols and organic acids [1]. As a famous traditional Chinese medicine (TCM), the tuber of G. elata is commonly used for the treatment of diverse mental diseases, involving insomnia [2, 3], depression [4-7], epilepsy, convulsion [8,9], and Alzheimer's disease [10, 11]. Plenty of pharmacological investigation suggested that G. elata had antihypertension [12], anti-tumor [13], anti-virus [14, 15] and anti-inflammation $[16,17]$ effects. Most of pharmacological components are 4-hydroxybenzyl alcohol, 4-hydroxybenzaldehyde, vanillin, 1,3-bis(4-hydroxybenzyl) citrate, 1-(4-beta-D-glucopyranosyloxybenzyl) citrate and parishin 
B [18]. However few reports about the psychoactive effects of polybenzyls were published [19-22]. In this study, the EtOAc extract of G. elata was found to activate $\mathrm{MT}_{2}$ receptor with an agonistic rate of $105.38 \%$ at the concentration of $102.22 \mu \mathrm{g} / \mathrm{mL}$. In order to characterize the active constituents, three new compounds (1-3, Fig. 1) were obtained by various column chromatography. Herein, we report their isolation, structural elucidation and agonistic activities on melatonin $\left(\mathrm{MT}_{1}\right.$ and $\left.\mathrm{MT}_{2}\right)$ receptors.

\section{Results and Discussion}

\subsection{Structure Elucidation}

Compound $\mathbf{1}$ had a molecular formula of $\mathrm{C}_{34} \mathrm{H}_{30} \mathrm{O}_{5}$ with 20 degrees of unsaturation, which was deduced from HRESIMS at $\mathrm{m} / \mathrm{z} 517.2040[\mathrm{M}-\mathrm{H}]^{-}$(calcd 517.2020). The UV absorptions at 281 and $254 \mathrm{~nm}$, and the IR absorptions at 3426 , $1631,1613,1511$ and $1439 \mathrm{~cm}^{-1}$ suggested the presence of hydroxyl and phenyl groups. In the ${ }^{1} \mathrm{H}-\mathrm{NMR}$ spectrum, three sets of protons $\left[\delta_{\mathrm{H}} 7.01(4 \mathrm{H}, \mathrm{d}, J=8.3 \mathrm{~Hz}, \mathrm{H}-2, \mathrm{H}-6\right.$, H-2 $\left.{ }^{\prime \prime \prime \prime}, \mathrm{H}-6^{\prime \prime \prime \prime}\right), 6.67$ (4H, overlap, H-3, H-5, H-3' $\left.{ }^{\prime \prime \prime \prime}, \mathrm{H}-5^{\prime \prime \prime \prime}\right)$; $6.93\left(2 \mathrm{H}, \mathrm{d}, J=8.4 \mathrm{~Hz}, \mathrm{H}-2^{\prime \prime \prime}, \mathrm{H}-6^{\prime \prime \prime}\right), 6.67$ (2H, overlap, $\left.\mathrm{H}-3^{\prime \prime \prime}, \mathrm{H}-5^{\prime \prime \prime}\right)$, and combining with ${ }^{1} \mathrm{H}-{ }^{1} \mathrm{H}$ COSY correlations of H-2/H-3, H-6/H-5, H-2,'"'/H-3' ${ }^{\prime \prime \prime}, \mathrm{H}-6^{\prime \prime \prime \prime} / \mathrm{H}-5^{\prime \prime \prime \prime}$, and of $\mathrm{H}-2^{\prime \prime \prime} / \mathrm{H}-3^{\prime \prime \prime}, \mathrm{H}-6^{\prime \prime \prime} / \mathrm{H}-5^{\prime \prime \prime}$ indicated the existence of 1,4 -substituted aromatic rings. One set of protons $\left[\delta_{\mathrm{H}} 6.85\right.$ (1H, brs, H-6"), $6.84\left(1 \mathrm{H}, J=8.4,2.0 \mathrm{~Hz}, \mathrm{H}-4{ }^{\prime \prime}\right), 6.67(1 \mathrm{H}$, overlap, $\left.\mathrm{H}-3^{\prime \prime}\right)$ ], and combining with ${ }^{1} \mathrm{H}-{ }^{1} \mathrm{H}$ COSY correlation of $\mathrm{H}-3$ " $/ \mathrm{H}-4$ " manifested the presence of a 1,2,4-substitued benzene ring. Besides, two meta-coupled protons $\left[\delta_{\mathrm{H}}\right.$ $6.77\left(1 \mathrm{H}, \mathrm{d}, J=2.0 \mathrm{~Hz}, \mathrm{H}-4^{\prime}\right)$ and $6.76(1 \mathrm{H}, \mathrm{d}, J=2.0 \mathrm{~Hz}$, H-6')], four methylenes [ $\delta_{\mathrm{H}} 3.79\left(4 \mathrm{H}, \mathrm{s}, \mathrm{H}-7, \mathrm{H}-7^{\prime \prime \prime}\right), 3.75$
(2H, s, H-7'), 3.68 (2H, s, H-7")] were well recognized (Table 1). Compared with 4-[[2-hydroxy-5-(methoxymethyl) phenyl] methyl]-2,6-bis [(4-hydroxyphenyl) methyl] phenol [19], compound 1 had an extra para- hydroxybenzyl [ $\delta_{\mathrm{H}} 6.93$

Table $1{ }^{1} \mathrm{H}-\mathrm{NMR}(400 \mathrm{MHz})$ and ${ }^{13} \mathrm{C}-\mathrm{NMR}(100 \mathrm{MHz})$ data of compound 1 in $\mathrm{CD}_{3} \mathrm{OD}$

\begin{tabular}{|c|c|c|c|c|}
\hline No. & $\delta_{\mathrm{H}}(J$ in $\mathrm{Hz})$ & $\delta_{\mathrm{C}}$ & No. $\delta_{\mathrm{H}}(J$ in $\mathrm{Hz})$ & $\delta_{\mathrm{C}}$ \\
\hline 1 & - & $133.6(\mathrm{~s})$ & $\begin{array}{l}\text { 3" } 6.67 \text { (1H, over- } \\
\text { lap) }\end{array}$ & $116.0(d)$ \\
\hline 2 & $7.01(1 \mathrm{H}, \mathrm{d}, 8.3)$ & $130.9(d)$ & $\begin{array}{l}4 " 6.84(1 \mathrm{H}, 8.4 \\
2.0)\end{array}$ & $128.5(\mathrm{~d})$ \\
\hline 3 & $\begin{array}{l}6.67(1 \mathrm{H}, \text { over- } \\
\text { lap) }\end{array}$ & 116.0 (d) & $5^{\prime \prime}-$ & $133.8(\mathrm{~s})$ \\
\hline 4 & - & $156.3(\mathrm{~s})$ & $6 " 6.85(1 \mathrm{H}, \mathrm{brs})$ & $132.2(\mathrm{~d})$ \\
\hline 5 & $\begin{array}{l}6.67(1 \mathrm{H}, \text { over- } \\
\text { lap) }\end{array}$ & 116.0 (d) & 7" $3.68(2 \mathrm{H}, \mathrm{s})$ & $41.3(\mathrm{t})$ \\
\hline 6 & $7.01(1 \mathrm{H}, 8.3)$ & $130.9(\mathrm{~d})$ & $1^{\prime \prime \prime}-$ & $134.5(\mathrm{~s})$ \\
\hline 7 & $3.79(2 \mathrm{H}, \mathrm{s})$ & $36.0(\mathrm{t})$ & $2^{\prime \prime \prime} 6.93(1 \mathrm{H}, \mathrm{d}, 8.4)$ & $130.8(\mathrm{~d})$ \\
\hline $1^{\prime}$ & - & $129.6(\mathrm{~s})$ & $\begin{array}{c}3^{\prime \prime \prime} 6.67(1 \mathrm{H}, \text { over- } \\
\text { lap) }\end{array}$ & $116.1(\mathrm{~d})$ \\
\hline $2^{\prime}$ & - & $154.2(\mathrm{~s})$ & $4^{\prime \prime \prime}-$ & $156.4(\mathrm{~s})$ \\
\hline $3^{\prime}$ & - & $129.5(\mathrm{~s})$ & $\begin{array}{c}5^{\prime \prime \prime} 6.67(1 \mathrm{H}, \text { over- } \\
\text { lap) }\end{array}$ & $116.1(d)$ \\
\hline $4^{\prime}$ & $6.77(1 \mathrm{H}, \mathrm{d}, 2.0)$ & $132.0(\mathrm{~d})$ & $6^{\prime \prime \prime} 6.93(1 \mathrm{H}, \mathrm{d}, 8.4)$ & $130.8(d)$ \\
\hline $5^{\prime}$ & - & $134.4(\mathrm{~s})$ & $7^{\prime \prime \prime} 3.79(2 \mathrm{H}, \mathrm{s})$ & $35.9(\mathrm{t})$ \\
\hline $6^{\prime}$ & $6.76(1 \mathrm{H}, \mathrm{d}, 2.0)$ & $132.0(d)$ & $1^{\prime \prime \prime \prime}-$ & $133.9(\mathrm{~s})$ \\
\hline $7^{\prime}$ & $3.75(2 \mathrm{H}, \mathrm{s})$ & $35.9(\mathrm{t})$ & $2^{\prime \prime \prime \prime} 7.01(1 \mathrm{H}, \mathrm{d}, 8.3)$ & $130.9(\mathrm{~s})$ \\
\hline $1^{\prime \prime}$ & - & $129.4(\mathrm{~s})$ & $\begin{array}{c}3^{\prime \prime \prime \prime} 6.67(1 \mathrm{H}, \text { over- } \\
\text { lap) }\end{array}$ & $116.0(\mathrm{~d})$ \\
\hline \multirow[t]{3}{*}{$2^{\prime \prime}$} & - & $154.1(\mathrm{~s})$ & $4^{\prime \prime \prime \prime}-$ & $154.2(\mathrm{~s})$ \\
\hline & & & $\begin{array}{c}5^{\prime \prime \prime \prime} 6.67(1 \mathrm{H}, \text { over- } \\
\text { lap) }\end{array}$ & $116.0(d)$ \\
\hline & & & $6^{\prime \prime \prime \prime} 7.01(1 \mathrm{H}, \mathrm{d}, 8.3)$ & $130.9(\mathrm{~d})$ \\
\hline
\end{tabular}

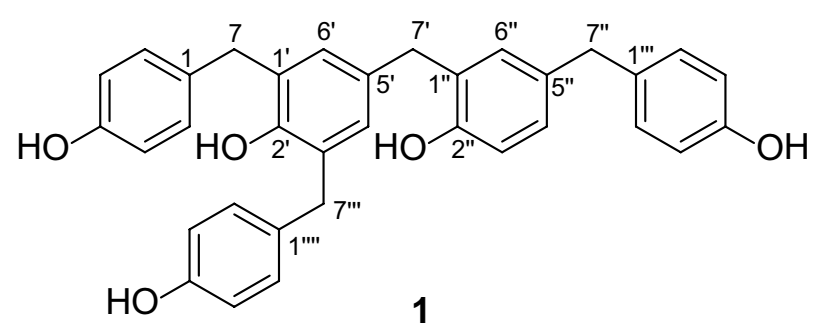

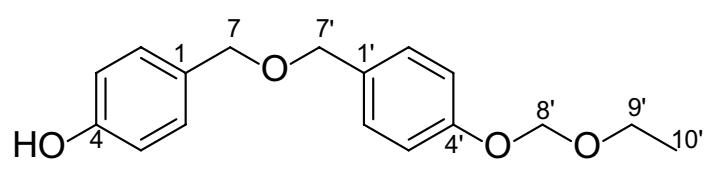

2<smiles>O=C([Te])Oc1ccc([Te]C([Te])c2ccc(O)cc2)cc1</smiles>

3

Fig. 1 Structures of compounds 1-3 
(2H, d, J = 8.4 Hz, H-2 $\left.{ }^{\prime \prime \prime}, \mathrm{H}-6^{\prime \prime \prime}\right), 6.67$ (2H, overlap, H-3"', H-5 $\left.{ }^{\prime \prime \prime}\right), 3.68$ (2H, s, H-7"); $\delta_{\mathrm{C}} 156.4$ (C-4"', s), 134.5 (C-1'"', s), 130.8 (C-2 $\left.2^{\prime \prime \prime}, \mathrm{C}-6^{\prime \prime \prime}, \mathrm{d}\right), 116.1$ (C-3 $\left.3^{\prime \prime \prime}, \mathrm{C}-5^{\prime \prime \prime}, \mathrm{d}\right), 41.3$ (C-7", t)], but with the absence of a methoxymethyl group. Based on the HMBC correlations from H-7" $\left(\delta_{\mathrm{H}} 3.68\right.$, s) to C-2"', C-6"' $\left(\delta_{\mathrm{C}} 132.1, \mathrm{~d}\right), \mathrm{C}-4$ " $(128.5, \mathrm{~d}), \mathrm{C}-6$ " $\left(\delta_{\mathrm{C}} 132.2\right.$, d), the linkages of C-7" to C-5" and C-1"' were established (Fig. 2). Thus, compound 1 was elucidated and named as gastropolybenzylol G.

Compound 2 was assigned a molecular formula of $\mathrm{C}_{17} \mathrm{H}_{20} \mathrm{O}_{4}$ with 8 degrees of unsaturation according to the HRESIMS at $\mathrm{m} / \mathrm{z} 333.1344$ [M+HCOO] $^{-}$(calcd for 333.1344). The UV (274, 247 and $226 \mathrm{~nm})$ and the IR (3423, 1613 and $1513 \mathrm{~cm}^{-1}$ ) absorptions showed the presence of hydroxyl and phenyl groups. Compared with 4,4'-hydroxybenzyl ether [23], compound $\mathbf{2}$ had an additional ethoxymethoxyl group $\left[\delta_{\mathrm{H}} 5.21\left(2 \mathrm{H}, \mathrm{s}, \mathrm{H}-8^{\prime}\right), 3.70\left(2 \mathrm{H}, \mathrm{q}, \mathrm{H}-9^{\prime}\right), 1.18\right.$ $\left(3 \mathrm{H}, \mathrm{t}, \mathrm{H}-10^{\prime}\right) ; \delta_{\mathrm{C}} 94.2\left(\mathrm{C}-8^{\prime}, \mathrm{t}\right), 65.2\left(\mathrm{C}-9^{\prime}, \mathrm{t}\right), 15.5$ (C-10', q)] in accordance with the HMBC correlation from $\mathrm{H}-8^{\prime}$ to C-9'. The HMBC correlation from $\mathrm{H}-8^{\prime}\left(\delta_{\mathrm{H}} 5.21,2 \mathrm{H}, \mathrm{s}\right)$ to $\mathrm{C}-4^{\prime}\left(\delta_{\mathrm{C}} 158.5, \mathrm{~s}\right)$ indicated the ethoxymethoxyl group at $\mathrm{C}-4^{\prime}$. Therefore, compound 2 was identified and named as gastropolybenzylol $\mathrm{H}$.

Compound 3 showed a molecular formula of $\mathrm{C}_{16} \mathrm{H}_{16} \mathrm{O}_{4}$ with 9 degrees of unsaturation according to the $[\mathrm{M}+\mathrm{H}]^{+}$ion at $\mathrm{m} / \mathrm{z} 273.1122$ (calcd for 273.1121). The UV absorptions at 275, 250 and $229 \mathrm{~nm}$ suggested the existence of phenolic structure and the IR absorptions at 3430, 1703, 1614 and $1518 \mathrm{~cm}^{-1}$ manifested the presence of hydroxyl, carbonyl and phenyl groups. Compared with 4,4'-hydroxybenzyl ether [23], compound $\mathbf{3}$ had an extra acetyl group [ $\delta_{\mathrm{C}} 172.9$ (s, C-8'), 21.0 (q, C-9')]. Thus, compound 3 was determined as the acetylated derivative of 4,4'-hydroxybenzyl ether and named as gastropolybenzylol I.

The known compounds were identified as 4, 4'-dimethoxy-dibenzyl ether [24] (4), 4,4'-diethoxybenzyl ether [25] (5), 4-[(4-(methoxymethyl) phenoxy] methyl] phenol [23] (6) and 4-[[4-[[4-(ethoxymethyl) phenoxy] methyl] phenoxy] methyl] phenol [26] (7).

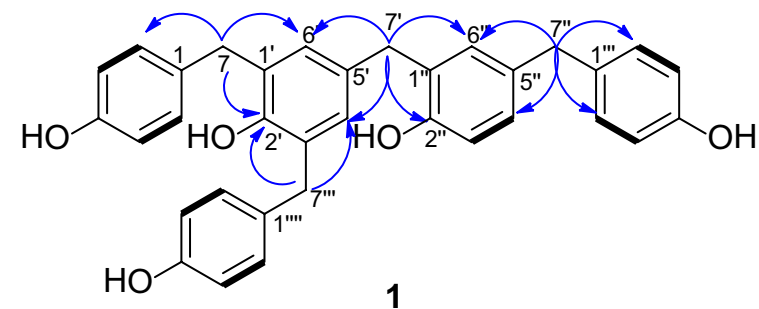

\section{2 $\mathrm{MT}_{1}$ and $\mathrm{MT}_{2}$ Receptors Agonistic Activities}

Compounds $\mathbf{1}$ and $\mathbf{2}$ were evaluated for their agonistic activities on $\mathrm{MT}_{1}$ and $\mathrm{MT}_{2}$ receptors in vitro. Melatonin was used as the positive control. As showed in Fig. 3, compound 1 could activate $\mathrm{MT}_{1}$ and $\mathrm{MT}_{2}$ receptors with agonistic rates of $55.91 \pm 4.84$ and $165.13 \pm 5.65 \%$ at the concentration of $0.5 \mathrm{mM}$. Due to 1 with better agonistic activities on $\mathrm{MT}_{2}$ receptor, further study provided an $\mathrm{EC}_{50}$ value of $76.24 \mu \mathrm{M}$ on $\mathrm{MT}_{2}$ receptor (Fig. 4).

\section{Experimental Section}

\subsection{General Procedures}

NMR spectra were undertaken by Avance III-400/III-600 spectrometers (Bruker, Bremerhaven, Germany). UV spectra were recorded on a UV-2401 equipment (Shimadzu, Kyoto, Japan). IR spectra were obtained on Bio-Rad FTS135 (Hercules, California, USA) spectrometers using $\mathrm{KBr}$

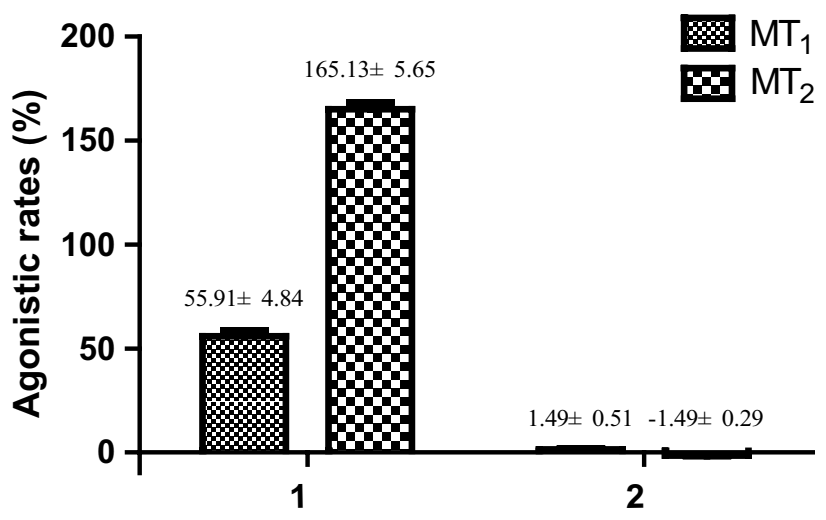

Fig. $3 \mathrm{MT}_{1}$ and $\mathrm{MT}_{2}$ receptors agonistic activities of compounds 1 and $2(0.5 \mathrm{mM})$. Data are expressed as mean \pm S.D., $n=3$. Melatonin was used as the positive control with $\mathrm{EC}_{50}$ values of $1.0 \mathrm{nM}\left(\mathrm{MT}_{1}\right)$ and $25.0 \mathrm{nM}\left(\mathrm{MT}_{2}\right)$
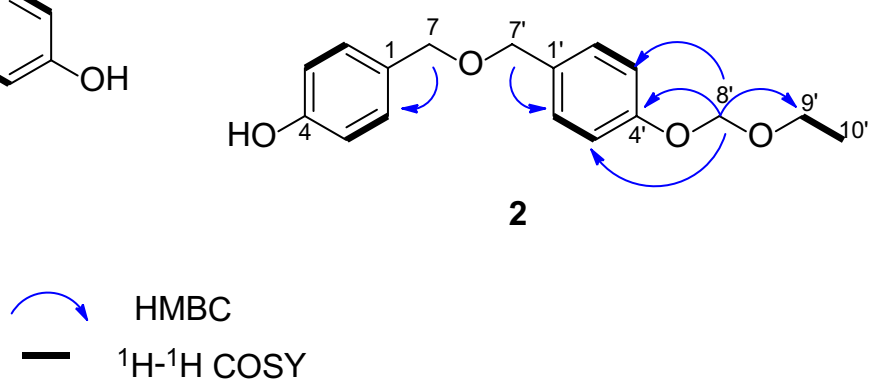

Fig. 2 The key ${ }^{1} \mathrm{H}_{-}{ }^{1} \mathrm{H}$ COSY and the HMBC correlations of compounds $\mathbf{1}$ and $\mathbf{2}$ 


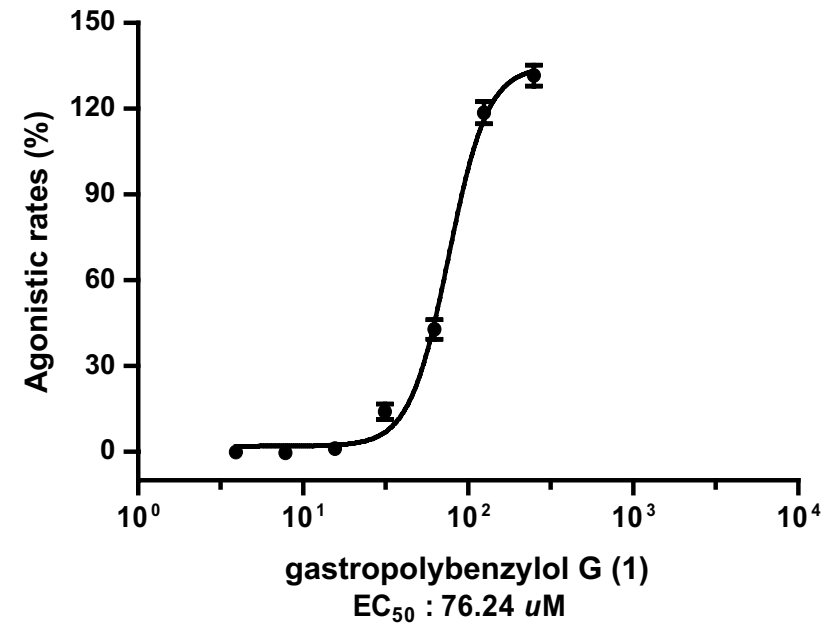

Fig. 4 Dose-dependent effects gastropolybenzylol G (1) on $\mathrm{MT}_{2}$ receptor

pellets. LCMS-IT-TOF (Shimadzu, Kyoto, Japan) was used for detecting MS spectra. Thin-layer chromatography (TLC) analyses were performed on silica gel GF254 plates (Jiangyou, Chemical Co. Ltd., Yantai, China). Compounds were purified by silica gel (200-300 mesh, Qingdao Makall group Co. Ltd., Qingdao, China), Sephadex LH-20 (Amersham Biosciences, Sweden) column chromatography and HPLC using Shimadzu LC-CBM-20 system (Shimadzu, Kyoto, Japan) with an Agilent XDB-C ${ }_{18}$ column $\left(9.4 \times 250 \mathrm{~mm}\right.$, Agilient, California, USA). $10 \% \mathrm{H}_{2} \mathrm{SO}_{4}$ in $\mathrm{EtOH}$ was applied for detecting spots by heating after sprayed. Melatonin as positive control was obtained from Damas-beta Co. Ltd. (Shanghai, China).

\subsection{Plant Material}

Gastrodia elata Bl. was bought from Zhaotong in Yunnan Province of China, in September 2014, and was identified by Prof. Li-Gong Lei, Kunming Institute of Botany, CAS. A voucher specimen (No. 20141107) was deposited at the Laboratory of Anti-virus and Natural Medicinal Chemistry, Kunming Institute of Botany, CAS.

\subsection{Extraction and Isolation}

Fresh rhizomes of $G$. elata $(45.0 \mathrm{~kg})$ were cut into slices and extracted with $90 \%$ aqueous ethanol $(45 \mathrm{~L} \times 3)$ at room temperature. The combined extract was concentrated under reduced pressure and partitioned between $\mathrm{H}_{2} \mathrm{O}$ and EtOAc. The EtOAc extract ( $88 \mathrm{~g}$ ) was separated by silica gel column chromatography (CC) using gradient elution with EtOAc$\mathrm{CHCl}_{3}(0: 100,5: 95,10: 90,20: 80,40: 60,100: 0, v / v)$ as the mobile phase to yield five fractions (Fr. 1-Fr. 5). Fr. 2 (22 g) was separated by silica gel CC with EtOAc-petroleum ether
$(5: 95,10: 90,20: 80,40: 60,100: 0)$ to yield five fractions (Fr. 2.1-Fr. 2.5). Then, Fr. 2.2 (3.4 g) was further separated by silica gel CC $\left(\mathrm{Me}_{2} \mathrm{CO}\right.$-petroleum ether, 3:97, 5:95, 10:90, 20:80, 50:50) to give five fractions (Fr. 2.2.1-Fr. 2.2.5). Compounds $2(10 \mathrm{mg})$ and $5(30 \mathrm{mg})$ were purified from Fr. 2.2.1 by HPLC on an Agilent $\mathrm{XDB}-\mathrm{C}_{18}$ column with the elution of $\mathrm{MeCN}-\mathrm{H}_{2} \mathrm{O}\left(85: 15, \mathrm{t}_{R}=30 \mathrm{~min}\right)$ and $\mathrm{MeCN}-\mathrm{H}_{2} \mathrm{O}$ (90:10, $\left.\mathrm{t}_{R}=27 \mathrm{~min}\right)$, respectively. Compound 4 (24 mg) was isolated from Fr. 2.3 by Sephadex $\mathrm{LH}-20\left(\mathrm{MeOH}-\mathrm{CHCl}_{3}\right.$, 50:50) and finally purified by HPLC with the mobile phase of $\mathrm{MeCN}-\mathrm{H}_{2} \mathrm{O}\left(60: 40, \mathrm{t}_{R}=23 \mathrm{~min}\right)$. Fr. 2.4 was purified from by Sephadex $\mathrm{LH}-20\left(\mathrm{MeOH}-\mathrm{CHCl}_{3}, 50: 50\right)$ and HPLC on an Agilent XDB- $\mathrm{C}_{18}$ column with the elution of $\mathrm{MeCN}$ $\mathrm{H}_{2} \mathrm{O}\left(60: 40, \mathrm{t}_{R}=20 \mathrm{~min}\right)$ to yield compound $3(3.5 \mathrm{mg})$. Fr. 3 was subjected to silica gel $\mathrm{CC}\left(\mathrm{Me}_{2} \mathrm{CO}\right.$-petroleum ether, 5:95, 10:90, 20:80, 40:60, 100:0) to yield five fractions (Fr. 3.1-Fr. 3.5). Fr 3.1 was isolated by silica gel CC $\left(\mathrm{Me}_{2} \mathrm{CO}-\mathrm{CHCl}_{3}, 5: 95,10: 90,20: 80,40: 60,100: 0\right)$, Sephadex $\mathrm{LH}-20 \mathrm{CC}\left(\mathrm{MeOH}-\mathrm{CHCl}_{3}, 50: 50\right)$, from which compounds $6\left(2 \mathrm{mg}, \mathrm{MeCN}-\mathrm{H}_{2} \mathrm{O}, 60: 40, \mathrm{t}_{R}=25 \mathrm{~min}\right)$ and 7 (37 mg, MeCN-H $\mathrm{H}_{2} \mathrm{O}, 45: 55, \mathrm{t}_{R}=20 \mathrm{~min}$ ) were obtained by HPLC purification. Further purification on Fr. 3.5 yielded compound 1 (10 mg) by silica gel CC, Sephadex LH-20 CC $\left(\mathrm{MeOH}-\mathrm{CHCl}_{3}\right.$ 50:50) and semi-preparative HPLC (MeCN$\left.\mathrm{H}_{2} \mathrm{O}, 30: 70, \mathrm{t}_{R}=40 \mathrm{~min}\right)$.

Gastropolybenzylol $G(1)$, pale yellow powder, UV $(\mathrm{MeOH}) \lambda_{\max }(\log \varepsilon): 281$ (4.03), 254 (3.25) nm; IR (KBr) $\nu_{\max }: 3426,1631,1613,1511,1439,1247,1229 \mathrm{~cm}^{-1}$; HRESIMS $m / z$ 517.2040 [M-H] ${ }^{-}$(calcd 517.2020). ${ }^{1} \mathrm{H}-$ NMR and ${ }^{13} \mathrm{C}-\mathrm{NMR}$ data showed in Table 1.

Gastropolybenzylol $H$ (2), yellow colloidal solid, UV $(\mathrm{MeOH}) \lambda_{\max }(\log \varepsilon): 275$ (3.38), $248(2.61), 226(4.30) \mathrm{nm}$; IR $(\mathrm{KBr}) \nu_{\max }: 3423,1613,1513,1223,1072,1003 \mathrm{~cm}^{-1}$; HRESIMS $m / z 333.1344[\mathrm{M}+\mathrm{COOH}]^{-}$(calcd 333.1344). ${ }^{1} \mathrm{H}-\mathrm{NMR}$ and ${ }^{13} \mathrm{C}-\mathrm{NMR}$ data showed in Table 2.

Gastropolybenzylol I (3), white powder, UV (MeOH) $\lambda_{\text {max }}$ ( $\log \varepsilon$ ): 275 (3.48), 250 (2.89), 229 (4.42) nm; IR (KBr) $\nu_{\max }: 3430,1703,1614,1518,1268,1245,1029,830 \mathrm{~cm}^{-1}$; HRESIMS $m / z 273.1117[\mathrm{M}+\mathrm{H}]^{+}($calcd 273.1121$) .{ }^{1} \mathrm{H}-$ NMR and ${ }^{13} \mathrm{C}-\mathrm{NMR}$ data showed in Table 2.

\subsection{Bioassay of Agonistic Activities on $\mathrm{MT}_{1}$ and $\mathrm{MT}_{2}$ Receptors}

Agonistic activities of compounds $\mathbf{1}$ and $\mathbf{2}$ were evaluated on HEK293 cell lines stably expressing the human melatonin $\mathrm{MT}_{1}$ and $\mathrm{MT}_{2}$ receptors. Referring to the previous study [27, 28], cells were cultivated by adding Dulbecco's modified eagle medium, G418 (400 $\mu \mathrm{g} / \mathrm{mL})$ and fetal bovine serum $(10 \%)$ in $5 \% \mathrm{CO}_{2}$ incubator at $37{ }^{\circ} \mathrm{C}$. Then, cells were put in a Matrigel ${ }^{\circledR}$ coated 96-well black plate at a density of $4 \times 10^{4}$ well and proliferated in $\mathrm{CO}_{2}$ for $24 \mathrm{~h}$. Wash Free Fluo-8 Calcium Assay Kit (HD Biosicences Co. 
Table $2{ }^{1} \mathrm{H}-\mathrm{NMR}$ and ${ }^{13} \mathrm{C}$ NMR data of compounds 2 and 3 in $\mathrm{CD}_{3} \mathrm{OD}$

\begin{tabular}{|c|c|c|c|c|}
\hline \multirow[t]{2}{*}{ No. } & \multicolumn{2}{|l|}{2} & \multicolumn{2}{|l|}{3} \\
\hline & $\delta_{\mathrm{H}}(500 \mathrm{MHz}, J$ in $\mathrm{Hz})$ & $\delta_{\mathrm{C}}(125 \mathrm{MHz})$ & $\delta_{\mathrm{H}}(600 \mathrm{MHz}, J$ in $\mathrm{Hz})$ & $\delta_{\mathrm{C}}(150 \mathrm{MHz})$ \\
\hline 1 & - & $130.2(\mathrm{~s})$ & - & $129.9(\mathrm{~s})$ \\
\hline 2 & $7.15(1 \mathrm{H}, \mathrm{d}, 8.6)$ & $130.9(d)$ & $7.17(1 \mathrm{H}, \mathrm{d}, 8.7)$ & $131.2(\mathrm{~d})$ \\
\hline 3 & $6.75(1 \mathrm{H}, \mathrm{d}, 8.6)$ & $116.1(d)$ & $6.85(1 \mathrm{H}, \mathrm{d}, 8.7)$ & $116.0(\mathrm{~d})$ \\
\hline 4 & - & $158.3(\mathrm{~s})$ & - & $160.5(\mathrm{~s})$ \\
\hline 5 & $6.75(1 \mathrm{H}, \mathrm{d}, 8.6)$ & $116.1(\mathrm{~d})$ & $6.85(1 \mathrm{H}, \mathrm{d}, 8.7)$ & $116.0(\mathrm{~d})$ \\
\hline 6 & $7.15(1 \mathrm{H}, \mathrm{d}, 8.6)$ & $130.9(d)$ & $7.17(1 \mathrm{H}, \mathrm{d}, 8.7)$ & $131.2(\mathrm{~d})$ \\
\hline 7 & $4.40(2 \mathrm{H}, \mathrm{s})$ & $72.8(\mathrm{t})$ & $4.92(2 \mathrm{H}, \mathrm{s})$ & $71.2(\mathrm{t})$ \\
\hline $1^{\prime}$ & - & $132.7(\mathrm{~s})$ & - & $129.4(\mathrm{~s})$ \\
\hline $2^{\prime}$ & $7.24(1 \mathrm{H}, \mathrm{d}, 8.7)$ & $130.6(d)$ & $7.15(1 \mathrm{H}, \mathrm{d}, 8.5)$ & $130.6(d)$ \\
\hline $3^{\prime}$ & $7.00(1 \mathrm{H}, \mathrm{d}, 8.7)$ & $117.2(\mathrm{~d})$ & $6.68(1 \mathrm{H}, \mathrm{d}, 8.5)$ & $116.3(\mathrm{~d})$ \\
\hline $4^{\prime}$ & - & $158.5(\mathrm{~s})$ & - & $158.6(\mathrm{~s})$ \\
\hline $5^{\prime}$ & $7.00(1 \mathrm{H}, \mathrm{d}, 8.7)$ & $117.2(\mathrm{~d})$ & $6.68(1 \mathrm{H}, \mathrm{d}, 8.5)$ & $116.3(\mathrm{~d})$ \\
\hline $6^{\prime}$ & $7.24(1 \mathrm{H}, \mathrm{d}, 8.7)$ & $130.6(d)$ & $7.15(1 \mathrm{H}, \mathrm{d}, 8.5)$ & $130.6(d)$ \\
\hline $7^{\prime}$ & $4.42(2 \mathrm{H}, \mathrm{s})$ & $72.4(\mathrm{t})$ & $4.85(2 \mathrm{H}, \mathrm{s})$ & $67.3(\mathrm{t})$ \\
\hline $8^{\prime}$ & $5.21(2 \mathrm{H}, \mathrm{s})$ & $94.2(t)$ & - & 172.9 (s) \\
\hline $9^{\prime}$ & $3.70(2 \mathrm{H}, \mathrm{q})$ & $65.2(\mathrm{t})$ & $1.94(3 \mathrm{H}, \mathrm{s})$ & $21.0(q)$ \\
\hline $10^{\prime}$ & $1.18(3 \mathrm{H}, \mathrm{t})$ & $15.5(q)$ & & \\
\hline
\end{tabular}

Ltd., Hd03-0010) was used for detecting calcium flow assay. Flex Station 3 Benchtop Multi-Mode Micro Plate Reader was applied for recording and reading data at room temperature using specified settings (excitation wave length, $485 \mathrm{~nm}$, emission wave length, $525 \mathrm{~nm}$, emission cut-off, $515 \mathrm{~nm}$ ). $\mathrm{EC}_{50}$ values were calculated by GraphPad Prism 5 software.

Acknowledgements This work was supported by the Program of Yunling Scholar, the Youth Innovation Promotion Association, CAS (2013252), and the Applied Basic Research Programs of Yunnan Province (2017FB137).

\section{Compliance with Ethical Standards}

Conflict of interest These authors have no conflict of interest to declare.

Open Access This article is distributed under the terms of the Creative Commons Attribution 4.0 International License (http://creativeco mmons.org/licenses/by/4.0/), which permits unrestricted use, distribution, and reproduction in any medium, provided you give appropriate credit to the original author(s) and the source, provide a link to the Creative Commons license, and indicate if changes were made.

\section{References}

1. L.M. Ojemann, W.L. Nelson, D.S. Shin, A.O. Rowe, R. Buchanan, Epilepsy Behav. 8, 376-383 (2006)

2. Y. Zhang, M. Li, R. Kang, J. Shi, G. Liu, J. Zhang, Pharmacol. Biochem. Behav. 102, 450-457 (2012)

3. Y. Shi, J.W. Dong, L.N. Tang, R.X. Kang, J.G. Shi, J.J. Zhang, Pharmacol. Biochem. Behav. 126, 146-151 (2014)
4. J.W. Jung, B.H. Yoon, H.R. Oh, J.H. Ahn, S.Y. Kim, S.Y. Park, J.F. Ryu, Biol. Pharm. Bull. 29, 261-265 (2006)

5. B.H. Zhou, X.J. Li, M. Liu, Z. Wu, X.M. Hu, Fitoterapia 77, 592-594 (2006)

6. S. Lin, W. Chen, K. Lu, P. Chen, S. Hsieh, T. Pan, S. Chen, L. Sheen, J. Agric. Food Chem. 62, 10493-10503 (2014)

7. P. Chen, C. Hsieh, K. Su, Y. Hou, H. Chiang, L. Sheen, Am. J. Chin. Med. 37, 1113-1124 (2009)

8. C. Hsieh, J. Lin, S. Chiang, S. Su, N. Tang, G. Lin, I. Lin, C. Liu, C. Hsiang, J. Chen, T. Ho, J. Ethnopharmacol. 109, 241-247 (2007)

9. J.H. Ha, D.U. Lee, J.T. Lee, J.S. Kim, C.S. Yong, J.A. Kim, J.S. Ha, K. Huh, J. Ethnopharmacol. 73, 329-333 (2000)

10. D.S.H.L. Kim, J. Kim, Y.S. Han, J. Altern. Complem. Med. 13, 333-340 (2007)

11. Y. Hu, C. Li, W. Shen, Neuropathology 34, 370-377 (2014)

12. O. Lee, K. Kim, C. Han, Y. Kim, H. Hong, Int. J. Mol. Sci. 13, 698-709 (2012)

13. J. Heo, S. Woo, M. Son, J. Park, W. Choi, K. Chang, S. Kim, E. Yoon, Y. Kim, H.M. Shin, S. Lee, Oncol. Rep. 18, 849-853 (2007)

14. H. Qiu, W. Tang, X. Tong, K. Ding, J. Zuo, Carbohydr. Res. 342, 2230-2236 (2007)

15. X. Tong, H. Qiu, X. Zhang, L. Shi, G. Wang, F. Ji, H. Ding, W. Tang, K. Ding, J. Zuo, Acta Pharmacol. Sin. 31, 585-592 (2010)

16. J.Y. Lee, Y.W. Jang, H.S. Kang, H. Moon, S.S. Sim, C.J. Kim, Arch. Pharm. Res. 29, 849-858 (2006)

17. S.M. Hwang, Y.J. Lee, D.G. Kang, H.S. Lee, Am. J. Chin. Med. 37, 395-406 (2009)

18. X.D. Yang, J. Zhu, R. Yang, J.P. Liu, L. Li, H.B. Zhang, Nat. Prod. Res. 21, 180-186 (2007)

19. .G. Shi, N.H. Chen, Y.N. Wang, Y.H. Yuan, S. Lin, M.M. Wu, Q.L. Guo, C.G. Zhu, China Patent CN103420805A, Dec 2013

20. J.Y. Huang, Y.H. Yuan, J.Q. Yan, Y.N. Wang, S.F. Chu, C.G. Zhu, Q.L. Guo, S.J. Gong, N.H. Chen, Acta Pharmacol. Sin. 37, 74-83 (2016) 
21. X.L. Zhang, Y.H. Yuan, Q.H. Shao, Z.Z. Wang, C.G. Zhu, J.G. Shi, K.L. Ma, X. Yan, N.H. Chen, Toxicol. Lett. 271, 74-83 (2017)

22. Q.H. Shao, X.L. Zhang, Y. Chen, C.G. Zhu, J.G. Shi, Y.H. Yuan, N.H. Chen, Mol. Immunol. 99, 115-123 (2018)

23. H. Taguchi, Y. Heihachiro, Y. Itiro, K. Yamasaki, I.H. Kim, Chem. Pharm. Bull. 29, 55-62 (1981)

24. S.G. Kim, K.N. Chung, S.B. Yang, J. Org. Chem. 52, 39173919 (1987)
25. J. Zhou, X.Y. Pu, Y.B. Yang, Sci. Bull. 18, 1118-1120 (1981)

26. Z.F. Li, Y.W. Wang, H. Ouyang, Y. Lu, Y. Qiu, Y. Feng, H. Jiang, X. Zhou, S. Yang, J. Chromatogr. B 988, 45-52 (2015)

27. Y.L. Zhang, L.H. Lai, S.M. Wang, Chin. J. Biotechnol. 25, 457463 (2009)

28. Y. Hasegawa, J.R. Erickson, G.J. Goddard, S.X. Yu, S.Y. Liu, K.W. Cheng, A. Eder, K. Bandoh, J. Aoki, R. Jarosz, A.D. Schrier, K.R. Lynch, G.B. Mills, X.J. Fang, J. Biol. Chem. 278, 1196211969 (2003) 